\title{
Use of health services by prison inmates: comparisons with the community
}

\author{
T Marshall, S Simpson, A Stevens
}

The future organisation of prison health care ${ }^{1}$ recommends that the NHS and the Prison Service work together to plan and provide health care services for prisoners. Whereas previously the Prison Service had this responsibility alone. The NHS will therefore have to take stock of the current level at which prison health care is demanded and provided. A key part of the information base for joint planning therefore includes data on current service utilisation. This paper compares primary care and inpatient health service utilisation data for UK prisoners with health services utilisation data for equivalent community populations.

In the community, primary care is provided principally by general practitioners and professions allied to medicine (mostly nurses). Residents in the community have access to the full range of NHS outpatient and inpatient facilities. Within prisons, primary care is provided by medical officers (some of whom are general practitioners), nursing grades and health care officers (who have received some training in health care). Some prisons have their own inpatient facilities (health care centre beds). These provide a low level of inpatient care and do not have a direct equivalent in the community. Prisoners also have access to NHS outpatient and inpatient facilities and to visiting specialists.

\section{Methods}

The prison population has a high turnover and varies from day to day. Because of this, the prison population is expressed as an average daily population during a one year period. The number of prisoners entering prisons-either as the result of new imprisonment or transfer between prisons-is expressed as the number of new receptions. Data on admissions to health care centre beds and NHS hospitals; numbers of consultations with primary health care workers; the average daily population of the prison estate; and the number of new receptions to prison were obtained from the Prison Service Directorate of Health Care. From this information consultation and admission rates per prisoner year were calculated. 18 January 2001
Some consultations are generated by the requirement that an assessment of the physical and mental health care needs of a prisoner is carried out on first reception into prison custody. ${ }^{2}$ An adjusted consultation rate, taking account of one consultation for each new reception, may therefore be a better reflection of service use. An adjusted consultation rate was therefore calculated, reducing the total number of consultations by one for each new reception.

The 1991/92 survey of morbidity in General Practice $^{3}$ provided age-sex specific consultation rates with general practitioners and nurses for the general population. Consultation rates were calculated for a population with the same demographic characteristics (age and sex structure) as the prison population. Data were also obtained on admission rates to NHS hospitals in England in 1996 and admission rates were calculated for a population with the same demographic characteristics as prison inmates. ${ }^{4}$

\section{Results}

PRIMARY CARE CONSULTATION RATES

Male prisoners consult doctors six times per prisoner year: three times more frequently than a demographically equivalent community population. They consult healthcare workers 23 times per prisoner year: 77 times more frequent than men in the community consult nurses (the nearest community equivalent to prison healthcare workers). Female prisoners consult doctors 14 times per prisoner year: three times more frequently than a demographically equivalent community population. They consult healthcare workers 59 times per prisoner year, 197 times more frequently than women in the community (table 1).

INPATIENT FACILITIES AND ADMISSION RATES In 1997/98, there were 1792 inpatient beds within prisons; 29 health care beds per 1000 prisoner years. In comparison, in 1996, there were approximately 4.5 beds per 1000 population in the UK as a whole. ${ }^{5}$ Prisoner admissions to NHS beds are lower than would be expected in a demographically equivalent community population (table 1). However, admissions to prison health care centre beds are high. Male prisoners are admitted to health care centre beds 0.7 times per prisoner year. Total inpatient admissions among male prisoners are 10 times those of a demographically equivalent community population. Female prisoners are admitted to health care centre beds over three times per prisoner year. Total inpatient admissions among female prisoners are 17 times those of a demographically equivalent community population. 


\section{Discussion}

Prison inmates are high users of primary care and of inpatient facilities compared with equivalent community populations. Possible explanations for this are an increased demand for health care-because of higher levels of morbidity and the institutional culture of prisons-and better access (supply) than is found in the community. Prisoners have a much higher incidence of mental health and substance misuse than the general population. However, increased morbidity alone is unlikely to explain the high service utilisation. ${ }^{6}$ There are other additional explanations that may be more open to change in the long run. Firstly, the legalistic aspect of the institutional culture of prisons requires prisoners to have medical consultations before adjudication proceedings, again adding to demand. Secondly, the institutional culture of prisons restricts prisoners' ability to self care and almost eliminates their access to over the counter medication. This allows little opportunity to substitute informal and self care for primary health care services. Institutional culture may also affect the threshold at which prisoners are admitted to prison inpatient facilities. It is as easy to admit patients to inpatient facilities as to arrange for self management with frequent review. Thirdly, on the supply side, prisoners have easy access to primary care for even trivial complaints. The personal cost and inconvenience of seeking professional care is therefore low. Even simple health problems therefore become medicalised. Similarly, compared with the community, prisons are relatively well supplied with inpatient facilities. Access is clearly also a factor in the lower than expected NHS hospital admission rate.

It is unlikely that morbidity differences between prisoners and a population of equivalent age and sex in the community is sufficient to explain differences in service use. But to determine whether this is the case data need to be collected on the reasons for prisoner consultations and reasons for inpatient admissions: data that are not currently collected. Meanwhile it seems reasonable to suggest that some loosening of legalistic reasons for prison
KEY POINTS

- Prisoners consult primary care doctors three times more frequently than equivalent community populations.

- Prisoners consult other primary healthcare workers almost 80 times more frequently than equivalent community populations.

- Prisoners receive inpatient care at least 10 times as frequently as equivalent community populations.

- The institutional culture of prisons, very restricted access to informal care (demand factors) and good access to formal health care (supply factors) are most likely factors explaining this high service use.

- Prison health care services could begin to tackle high utilisation rates by reducing legalistic reasons for consultation and increasing prisoners' ability to self care.

health service use and greater allowance for self care could be useful starting points for reducing demands on prison health care services.

Contributors

Tom Marshall and Sue Simpson obtained and collated data from the different sources and contributed to writing the paper. Andrew Stevens was involved at the inception and contributed to writing the paper.

Funding: none.

Conflicts of interest: none.

1 Joint Prison Service and National Health Service Executive Working Group. The future organisation of prison health care. London: Department of Health, 1999

2 HM Prison Service Health Care Directorate. Health care standards for prisons in England and Wales. London: Prison Service, 1994.

3 McCormick A, Fleming D, Charlton J. Morbidity statistics from general practice. Fourth national study, 1991-1992. London: HMSO, 1995.

4 Department of Health. Hospital episode statistics, England 1995/96. London: Department of Health.

5 Organisation for Economic Co-operation and Development. OECD health data 1998. CD-Rom. Paris: 1998.

6 Singleton N, Meltzer H, Gatward R, et al. Psychiatric morbidity among prisoners in England and Wales: The report of a survey carried out in 1997 by Social Survey Division Of the Office for National Statistics on behalf of the Department of Health. London: The Stationery Office, 1998. 